\title{
Risk Factors for Major Amputation in Diabetic Foot Ulcer Patients
}

\author{
Qingwei Lu $\mathbb{D}^{\prime}$ \\ Jun Wang' \\ Xiaolu Wei ${ }^{2}$ \\ Gang Wang' \\ Yang $\mathrm{Xu}{ }^{\prime}$ \\ 'Department of Ulcers and Peripheral \\ Vascular Surgery, First Teaching Hospital \\ of Tianjin University of Traditional \\ Chinese Medicine, National Clinical \\ Research Center for Chinese Medicine \\ Acupuncture and Moxibustion, Tianjin, \\ 300193, People's Republic of China; \\ ${ }^{2}$ School of Integrated Traditional Chinese \\ and Western Medicine, Binzhou Medical \\ University, Yantai, 264003, People's \\ Republic of China
}

Background: The purpose of our study was to identify the new and the more specific risk factors for major amputation in patients with diabetic foot ulcer (DFU).

Methods: We used data from the Electronic Medical Record (EMR) database of our hospital from February 2014 to July 2020. Patients with DFU and amputation were included in the study. The logistic regression model was adjusted for demographic characteristics and related comorbidities between major and minor amputation groups.

Results: Among 3654 patients with DFU, 363 (9.9\%) were amputated. Patients with major versus minor amputation, in multivariable logistic regression models, major amputation independent factors included previous amputation history (odds ratio [OR] 2.31 [95\% CI $1.17-4.53], p=0.02)$, smoking (2.58 [1.31-5.07], $p=0.01)$, coronary artery disease (CAD) (2.67 [1.35-5.29], $p=0.03)$, ankle brachial index $(\mathrm{ABI})<0.4(15.77$ [7.51-33.13], $p<0.01)$, Wagner 5 (5.50 [1.89-16.01], $p<0.01)$, activated partial thromboplastin time (APTT) (1.23 [1.03-1.48], $p=0.01)$, glycosylated hemoglobin A1c (HbA1c) $(1.23$ [1.03-1.48], $p=0.03)$, hemoglobin $(\mathrm{Hb})(0.98$ [0.96-1.00], $p=0.01)$, plasma albumin (ALB) $(0.88$ [0.81-0.95], $p<$ $0.01)$ and white blood cell (WBC) (1.10 [1.04-1.16], $p<0.01)$.

Conclusion: Major amputation was associated with previous amputation history, smoking, CAD, Wagner 5, ABI <0.4, HbA1c, Hb, ALB, WBC, and APTT might be a new independent factor. In view of these factors, early prevention and guidance promptly orientated by multidisciplinary is of great significance to reduce the disability rate and economic burden. Keywords: diabetic foot ulcers, risk factors, major amputation, minor amputation, retrospective analysis

\section{Introduction}

The prevalence of diabetes mellitus (DM) is rising significantly, and the World Health Organization has reported that the estimated number of patients with diabetes was nearly 425 million in 2017 , consequently, increasing the number of diabetes-related complications. ${ }^{1}$ Diabetes prevalence increases with age so the highest estimated prevalence is in people older than 65. In 2019, the estimated number of people with diabetes aged 65-99 years is 135.6 million (19.3\%). If this trend continues, the number of people above 65 years (65-99 years) with diabetes will be 195.2 million in 2030 and 276.2 million in $2045 .^{2}$ Chronic diabetic complications are a serious health concern as well as an economic burden. ${ }^{3}$ Among complications, it is generally known that diabetic foot ulcer (DFU) is the most frequently recognized complication, which is a kind of disease related to neuropathy and/or peripheral arterial disorder of the lower extremities and with infection, ulceration, and destruc tion of deep tissues in diabetic patients, as a result
Correspondence: Jun Wang; Gang Wang Department of Ulcers and Peripheral Vascular Surgery, First Teaching Hospital of Tianjin University of Traditional Chinese Medicine, National Clinical Research Center for Chinese Medicine Acupuncture and Moxibustion, Tianjin, 300000, People's Republic of China Email tjzywangjun@126.com; doctorwanggang@sina.com 
of the interaction of factors induced by sustained and uncontrolled hyperglycemia. ${ }^{4}$ DFU is a rising health problem due to increasing prevalence of diabetes worldwide, and treatment of these foot ulcers is challenging because of their multifactorial aetiology, and it places a high burden on patients, healthcare systems and society. ${ }^{5}$ It is estimated that $15-25 \%$ of people with diabetes will be affected by a foot ulcer at some point in their lives. ${ }^{1,6}$ Recurrence of DFU is also common, with reported rates of $30-40 \%$ within 1 year after DFU healing. ${ }^{7}$ The risk of death at 5 years for a patient with a DFU is 2.5 times as high as the risk for a patient with diabetes who has no foot ulcer. $^{8}$ Therefore, prevention of foot ulcers is of paramount importance and has long been recognized as a priority by the International Working Group on the Diabetic Foot (IWGDF). Perhaps the most unpleasant potential consequence of DFU besides death is lower extremity amputation (LEA).

Amputation related diabetes is various in different countries. The annual incidence of amputation is $0.3 \%$ in the US and Japan, ${ }^{9} 0.3 \%$ in Ireland, ${ }^{10} 0.6 \%$ in the Netherlands, $^{11}$ and $0.6 \%$ in Mexican and non-Mexican Americans. ${ }^{12}$ The incidence rate of amputation is greatly increased when the ulcer of diabetic patients occurs. The annual incidence and 7-year average incidence of amputation for DFU patients were 5.1 and $9.9 \%$ respectively in China. ${ }^{4,13}$ Surprisingly, amputation rate as high as $52 \%$ was reported among patients hospitalized for DFU in one tertiary healthcare center in Nigeria. ${ }^{14}$ Efforts to prevent this unpleasant situation therefore deserve utmost attention, and this could be partly accomplished by risk factor identification.

It is widely known that amputation includes major amputation and minor amputation. This type treatment of amputation, undoubtedly, severely limits personal functional capacity, especially in fragile individuals. ${ }^{15}$ However, patients with major amputation have more pain and poorer social function than those with minor amputation. Conversely, patients with minor amputation are more independent, ambulatory and have better quality of life than those with major amputation. ${ }^{16}$ Therefore, identification of risk factors for major amputation is crucial for the prognosis of patients with DFU.

Several studies reported the risk factors for amputation of patients with DFU in recent years. ${ }^{17-21}$ Unfortunately, few studies compared data between major and minor amputations. This study seeked to identify the more specific risk factors associated with major amputations so that we can develop early management strategies, reduce the incidence rate of amputation and improve treatment effect of patients with DFU.

\section{Materials and Methods}

We reviewed the electronic medical record (EMR) database of our hospital from February 2014 to July 2020. The EMR database was queried using national clinical version 2.0 disease diagnosis code (ICD-10) and the hospital discharge diagnostic code E14.500×050 was defined as inclusion criteria. This code represented the patient's diagnosis of DFU. Finally, 3,654 patients who were diagnosed as having DFU were identified in the study. Patients with amputation (major and minor) were selected from the above patients for grouping analysis.

We reviewed patients' age, gender, smoking history, diabetes duration, ulcer duration, Wagner classification, ankle brachial index (ABI), previous amputation history, multi-drug resistant bacterial infection (MDR), white blood cell (WBC), plasma albumin (ALB), hemoglobin $(\mathrm{Hb})$, glycosylated hemoglobin A1c (HbAlc), D-Dimer, fibrinogen (Fib), activated partial thromboplastin time (APTT), and medical comorbidities, including hypertension, hyperlipidemia, coronary artery disease (CAD), peripheral arterial disease (PAD), chronic renal insufficiency (CRI), sequelae of cerebral infarction.

Major amputation, ie, above-the-ankle amputation, amputation below the ankle level is considered a minor amputation. Smoking history includes present and past situations. ABI less than 0.4 is defined as severe ischemia, 0.4-0.7 as moderate ischemia, and $0.7-0.9$ as mild ischemia. Previous amputation history is defined as the amputation history before hospitalization, including major amputation and minor amputation. MDR are resistant to three or more kinds of antibiotics at the same time. PAD and CAD are diagnosed by Doppler ultrasonography and the left ventricular ejection fraction (EF) of patients with CAD was less than $60 \%$. Sequelae of cerebral infarction includes the patients with limb dysfunction after ischemic or hemorrhagic stroke. In addition, the patients were documented to have CRI if their baseline creatinine was $>1.5 \mathrm{mg} / \mathrm{dl}$ or glomerular filtration rate $<60 \mathrm{~mL} / \mathrm{min}$.

The data were subjected to frequency analysis, to obtain the baseline characteristics of all patients. We compared patients' demographics and other parameters between patients who had major amputation (major amputation group) with those who had minor amputation (minor amputation group). Student' $t$-test was used for the 
continuous parameters and the chi-square test for categorical parameters. Statistical significance was determined at two-tailed $p<0.05$.

Risk factors of major lower limb amputation were determined using univariate logistic regression analysis, risk factors with $p<0.1$ were selected for multivariate logistic regression analysis. The variables with $p<0.05$ were independent risk factors for major amputation. Odds ratios (ORs) with corresponding 95\% confidence intervals $(95 \% \mathrm{CI})$ were calculated and the forest maps of risk factors were drawn. Multicollinearity and interaction were checked and were not found, indicating each variable was independent.

The data exported from the EMR system were analyzed statistically and received in the form of Microsoft Excel 2007 files. Then, the statistical analysis was conducted by using IBM SPSS v.21 statistical software, and the figure was drawn with GraphPad Prism v.8 graphics software.

\section{Results}

This study included the data of 3,654 consecutive patients, who were hospitalized for the management of DFU at the diabetic wound center of First Teaching Hospital of Tianjin University of Traditional Chinese Medicine from 28 February 2014 to 8 July 2020. 363 patients (363/3654, 9.9\%) developed LEA (major and minor), 139 cases in major amputation group and 224 cases in minor amputation group (Figure 1). Patients who were readmitted because of recurrent ulcers were not excluded in the study. There were significant differences in age, gender, diabetes duration, MDR bacterial infection, hypertension, hyperlipidemia, cerebral infarction, renal insufficiency between the two groups $(p>0.05$, Table 1$)$.

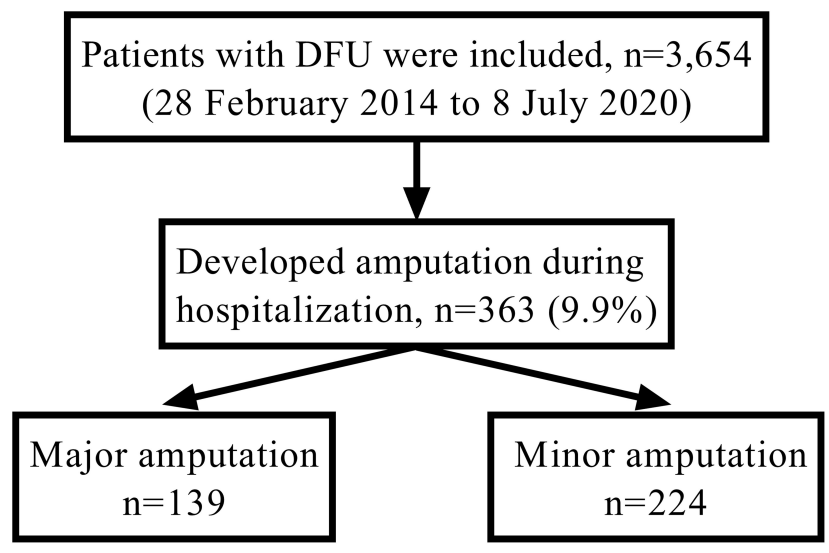

Figure I Selection flow diagram.

\section{Univariate Analysis of Risk Factors for Major Amputation}

In the univariate analysis, fourteen variables had significant difference between major amputation group and minor amputation group in the study (Table 1). Smoking history (odds ratio [OR] 1.78 [95\% CI 1.14-2.77], $p=$ $0.01)$, ulcer duration (1.08 [1.02-1.14], $p=0.01)$, Wagner 4 (2.13 [1.12-4.07], $p=0.02)$, Wagner 5 (4.30 [2.188.48], $p<0.01), \mathrm{ABI}<0.4$ (8.22 [5.02-13.46], $p<0.01)$, previous amputation (2.56 [1.61-4.06], $p<0.01)$, WBC (1.17 [1.12-1.22], $p<0.01)$, ALB (0.82 [0.78-0.86], $p<$ $0.01), \mathrm{Hb}(0.96$ [0.95-0.97], $p<0.01), \mathrm{HbAlc}(1.23$ [1.09-1.38], $p<0.01)$, D-Dimer (1.26 [1.04-1.52], $p=$ $0.01)$, Fib (1.33 [1.17-1.50], $p<0.01)$, APTT (1.07 [1.04-1.10], $p<0.01)$, CAD (1.74 [1.11-2.71], $p=0.02)$ and PAD (1.96 [1.19-3.22], $p=0.01)$ were included in stepwise multivariate logistic regression analysis.

\section{Multivariate Analysis of Risk Factors for Major Amputation}

To investigate independent risk factors of major amputation, stepwise multivariate logistic regression analysis was performed. We found that major amputation was associated with previous amputation history (odds ratio [OR] $2.31[95 \%$ CI 1.17-4.53], $p=0.02)$, smoking (2.58 [1.31-5.07], $p=0.01), \mathrm{CAD}(2.67[1.35-5.29], p=$ $0.03), \mathrm{ABI}<0.4$ (15.77 [7.51-33.13], $p<0.01)$, Wagner 5 (5.50 [1.89-16.01], $p<0.01)$, APTT (1.23 [1.03-1.48], $p=0.01), \mathrm{HbA} 1 \mathrm{c}(1.23$ [1.03-1.48], $p=0.03), \mathrm{Hb}(0.98$ [0.96-1.00], $p=0.01)$, ALB (0.88 [0.81-0.95], $p<0.01)$ and WBC (1.10 [1.04-1.16], $p<0.01)$ (Table 2). The results were visually revealed by forest plots (Figure 2).

\section{Discussion}

DFU is a significant health care problem, patients and families incur substantial economic burden for society. Risk factors for diabetic foot major amputation have been intensively studied during the last decades, while, the risk factors in previous studies were various. Such variability outcomes might be due to variations study designs and different clinical characteristics of the population. In this study, we aimed to investigate the clinical and biochemical factors associated with major amputation in patients with DFU. We considered that through the study of major amputation group and minor amputation group in patients with diabetic foot, the specificity of the risk factors of major amputation was higher than other grouping 
Table I Clinical Characteristics and Univariate Analysis of Risk Factors for Patients with Major Amputation and Minor Amputation

\begin{tabular}{|c|c|c|c|c|c|}
\hline Characteristic/Variable & Major Amputation Group $(n=139)$ & Minor Amputation Group $(n=224)$ & $p$ value & OR & $95 \% \mathrm{Cl}$ \\
\hline Age, years & $65.68 \pm I 1.00$ & $65.24 \pm 10.85$ & 0.71 & & \\
\hline Age & & & 0.38 & & \\
\hline$<45$ & $9(6.5)$ & II (4.9) & 0.39 & & \\
\hline $46-55$ & $12(8.6)$ & $35(15.6)$ & 0.12 & & \\
\hline $56-65$ & $45(32.4)$ & $67(22.9)$ & 0.69 & & \\
\hline $66-75$ & $50(36.0)$ & $69(30.8)$ & 0.80 & & \\
\hline $76-85$ & $20(14.4)$ & $39(17.4)$ & 0.38 & & \\
\hline$>85$ & $3(2.2)$ & $3(1.3)$ & 0.83 & & \\
\hline Gender & & & 0.10 & & \\
\hline Female & $34(24.5)$ & $73(32.6)$ & & & \\
\hline Male & $105(75.5)$ & $151(67.4)$ & & & \\
\hline Smoking (present or past) & $94(67.6)$ & $|2|(54)$ & 0.01 & 1.78 & I. $14-2.77$ \\
\hline Diabetes duration, years & $15.22 \pm 6.64$ & $15.53 \pm 8.43$ & 0.71 & & \\
\hline Ulcer duration, months & $4.35 \pm 6.00$ & $2.82 \pm 3.52$ & 0.01 & 1.08 & I.02-I.14 \\
\hline MDR bacterial infection & $79(61.2)$ & $137(56.8)$ & 0.44 & & \\
\hline Wagner classification & & & 0.00 & & \\
\hline 3 & $15(10.8)$ & $57(25.4)$ & & & \\
\hline 4 & $64(46.0)$ & II 4(50.9) & 0.02 & 2.13 & I.12-4.07 \\
\hline 5 & $60(43.2)$ & $53(23.7)$ & 0.00 & 4.30 & $2.18-8.48$ \\
\hline$A B I$ & & & 0.00 & & \\
\hline$\geq 0.4$ & $54(38.8)$ & 188(83.9) & & & \\
\hline$<0.4$ & $85(6 \mid .2)$ & $36(16.1)$ & 0.00 & 8.22 & $5.02-13.46$ \\
\hline Previous amputation history & $58(4 I .7)$ & $49(21.9)$ & 0.00 & 2.56 & $1.6 I-4.06$ \\
\hline WBC, $\times 10^{9} / \mathrm{L}$ & $|5.2| \pm 8.22$ & $9.79 \pm 4.52$ & 0.00 & 1.17 & $1.12-1.22$ \\
\hline ALB, g/L & $28.91 \pm 4.56$ & $33.63 \pm 5.83$ & 0.00 & 0.82 & $0.78-0.86$ \\
\hline $\mathrm{Hb}, \mathrm{g} / \mathrm{L}$ & $96.03 \pm 19.16$ & $111.30 \pm 20.95$ & 0.00 & 0.96 & $0.95-0.97$ \\
\hline $\mathrm{HbAlc} \%$ & $8.80 \pm 1.85$ & $8.10 \pm 1.82$ & 0.00 & 1.23 & $1.09-1.38$ \\
\hline D-Dimer & $1.32 \pm 1.50$ & $0.98 \pm 1.01$ & 0.01 & 1.26 & $1.04-1.52$ \\
\hline Fib, g/L & $6.38 \pm 1.79$ & $5.43 \pm 1.83$ & 0.00 & 1.33 & $1.17-1.50$ \\
\hline APTT, second & $36.32 \pm 9.19$ & $32.04 \pm 7.33$ & 0.00 & 1.07 & $1.04-1.10$ \\
\hline \multicolumn{6}{|l|}{ Comorbidities } \\
\hline hypertension & $82(59.0)$ & $149(66.5)$ & 0.18 & & \\
\hline hyperlipidemia & $17(12.2)$ & $22(9.8)$ & 0.49 & & \\
\hline CAD & $95(68.3)$ & $124(55.4)$ & 0.02 & 1.74 & $|| 1-2.7 \mid$. \\
\hline PAD & III(79.9) & $150(67.0)$ & 0.01 & 1.96 & I.19-3.22 \\
\hline Cerebral infarction & $4 I(29.5)$ & $74(33.0)$ & 0.56 & & \\
\hline Renal insufficiency & $20(14.4)$ & $4 I(18.3)$ & 0.33 & & \\
\hline
\end{tabular}

Notes: Continuous variables are presented as mean and standard deviation $(M \pm S D)$; categorical parameters are presented as counts, with percentages in parentheses, $n(\%)$. Abbreviations: OR, odds ratio; $\mathrm{Cl}$, confidence interval; MDR, multi-drug resistant; $\mathrm{ABI}$, ankle brachial index; WBC, white blood cell; $\mathrm{ALB}$, plasma albumin; $\mathrm{Hb}$, hemoglobin; $\mathrm{HbAlc}$, glycosylated hemoglobin; Fib, fibrinogen; APTT, activated partial thromboplastin time; CAD, coronary artery disease; PAD, peripheral arterial disease; CRI, chronic renal insufficiency. 
Table 2 Multivariate Analysis of Risk Factors for Patients with Major Amputation and Minor Amputation

\begin{tabular}{|c|c|c|c|}
\hline Variable & $p$ value & OR & $95 \% \mathrm{Cl}$ \\
\hline Smoking (present or past) & 0.01 & 2.58 & $1.31-5.07$ \\
\hline Ulcer duration, months & 0.13 & & \\
\hline Wagner classification & 0.01 & & \\
\hline 3 & & 1 & \\
\hline 4 & 0.06 & & \\
\hline 5 & $<0.01$ & 5.50 & $1.89-16.01$ \\
\hline$A B I$ & $<0.01$ & & \\
\hline$\geq 0.4$ & & 1 & \\
\hline$<0.4$ & $<0.01$ & 15.77 & $7.51-33.13$ \\
\hline Previous amputation history & 0.02 & 2.31 & $1.17-4.53$ \\
\hline WBC, $\times 10^{9} / \mathrm{L}$ & $<0.01$ & 1.10 & $1.04-1.16$ \\
\hline ALB, $g / L$ & $<0.01$ & 0.88 & $0.8 I-0.95$ \\
\hline $\mathrm{Hb}, \mathrm{g} / \mathrm{L}$ & 0.01 & 0.98 & $0.96-1.00$ \\
\hline $\mathrm{HbAlc}, \%$ & 0.03 & 1.23 & $1.03-1.48$ \\
\hline D-Dimer & 0.81 & & \\
\hline Fib, g/L & 0.48 & & \\
\hline APTT, second & 0.01 & 1.05 & $1.01-1.09$ \\
\hline CAD & 0.01 & 2.67 & $1.35-5.29$ \\
\hline PAD & 0.97 & & \\
\hline
\end{tabular}

Abbreviations: $\mathrm{OR}$, odds ratio; $\mathrm{Cl}$, confidence interval; $\mathrm{ABI}$, ankle brachial index; WBC, white blood cell; ALB, plasma albumin; $\mathrm{Hb}$, hemoglobin; HbAlc, glycosylated hemoglobin; Fib, fibrinogen; APTT, activated partial thromboplastin time; CAD, coronary artery disease; PAD, peripheral arterial disease.

methods. In our study, amputation rate was $9.9 \%$, which was similared to the data reported by other studies. ${ }^{22}$ Finally, the multivariate stepwise logistic regression analysis showed that ten items were risk factors for major amputation.

There was no difference between major amputation and minor amputation groups with respect to the duration of diabetes and ulcer in our study. Regarding the influence of HbA1c level, previous researches reported conflicting results. Yesil et al found that diabetes duration and $\mathrm{HbAlc}$ were not risk factors predicting overall amputations in patients with DFU. ${ }^{23}$ However, HbAlc was a risk factor of major amputation in our study, and Moon et al also proved this conclusion. ${ }^{24} \mathrm{HbAlc}$ reflected the level of fasting blood glucose in diabetic patients in recent 3 months. Previous studies also showed that poor diabetes control was a risk factor for limb loss in diabetic patients. $^{25}$ Therefore, good glycemic control is essential for patients with DFU to prevent the progression of the disease and reduce the risk to be amputated.

Amputation history reflected the progression of patients with DFU. Miller's study held that patients who have minor amputation were at increased risk for belowthe-knee amputation. ${ }^{26}$ Besides, 1,873 patients who underwent amputation were investigated in a meta-analysis, ${ }^{27}$ which drew the conclusion that the risk of re-amputation in patients with amputation history was 1.47 -fold that in patients without amputation history. The above conclusions were similar to our study. LEA caused a change in the biomechanics of the amputated limb and potentially creates higher pressure areas and deformities. Patients with previous major amputations rely exclusively on the contralateral limb for ambulation, thus making the limb more susceptible to trauma, recurrent ulceration and LEA. Therefore, for patients with DFU who have been
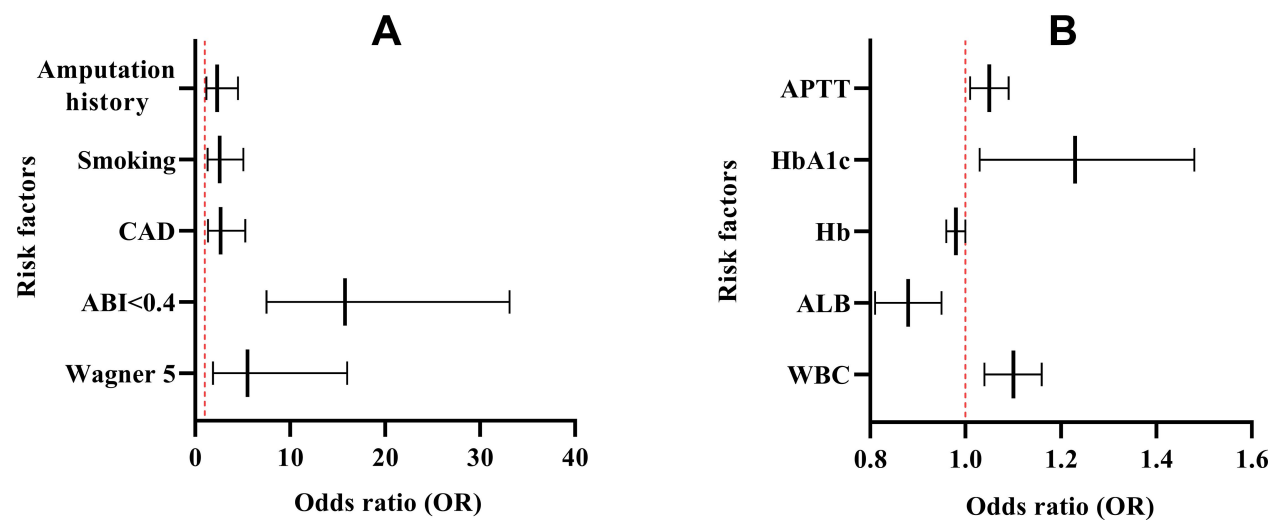

Figure 2 Independent risk factors for major amputation. (A) categorical variable (amputation history, smoking, CAD, ABI<0.4, Wagner 5); (B) continuous variables (APTT, HbAlc, Hb, ALB, WBC).

Abbreviations: OR, odds ratio; $\mathrm{Cl}$, confidence interval; $\mathrm{CAD}$, coronary artery disease; $\mathrm{ABI}$, ankle brachial index; APTT, activated partial thromboplastin time; HbAlc, glycosylated hemoglobin; Hb, hemoglobin; ALB, plasma albumin; WBC, white blood cell. 
amputated (major or minor), preventive measures should be taken in advance according to the risk factors to reduce the probability of re-amputation.

Smoking was deduced as a risk factor in this study, by contrary, hypertension and hyperlipidemia were not risk factors for LEA of patients with DFU. A meta-analysis shown that smoking was associated with DFU amputation ([OR] 1.65 [95\% CI] 1.09-2.50) and smoking cessation served as a protective measure against DF amputation. ${ }^{28}$ But, different conclusion from our study was that hypertension and hyperlipidemia were all risk factors in other studies. $^{29,30}$ These factors are easy to lead to atherosclerosis, progressive PAD, which increasing peripheral ischemia, blood flow will be blocked, and ultimately make DFU difficult to heal. Considering the combination of increased atherothrombotic risk and the risk of microvascular disease, patients with diabetes have a higher risk of lower limb ischemia and amputation. In addition, smoking and PAD could increase thirty-day readmission rates following primary LEA in patients with DFU, the OR with $95 \%$ CI were (3.22 [1.40 7.36]) and (2.47 [1.08 5.67]) respectively in Ries's study. ${ }^{31}$ It was universally known that limb ischemia has also been identified as an independent risk factor for amputation in patients with diabetic foot lesions. Calle-Pascual's study showed that all major amputations patients had peripheral vascular disease. ${ }^{32}$ Our study showed that the incidence of PAD in patients with major amputation was 67.0\% (150/224). Although PAD was not an independent risk factor in this study, ABI could better reflect the degree of lower limb ischemia. The study has shown that when $\mathrm{ABI}<0.4$, ie severe ischemia, the risk probability of major amputation factor in patients with DFU were 15.77-fold higher than those in patients with mild-moderate ischemia. Faglia et al found a significant increase in major amputation at 30 days and 5 years in a diabetic patient population with critical limb ischemia that did not have revascularization after presentation. ${ }^{33}$ These demonstrated the importance of assessing the peripheral vascular status in all diabetic patients with DFU. Early referral to a vascular surgeon might delay (or prevent) major LEA.

In patients with $\mathrm{CAD}$, heart pumping function was weakened, lower limb ischemia and hypoxia were aggravated, which increased the risk of amputation, and even increased the risk of death. ${ }^{34}$ We found that CAD significantly increased the risk of major amputation, fortunately, none of the patients died during hospitalization. A cohort study in Japan showed that CAD was associated with 2.5-fold and 1.8-fold higher risk of PAD and amputation, respectively, among patients without diabetes, and associated with 3.9-fold and 9.5-fold higher risk of PAD and lower limb amputation among patients with diabetes. ${ }^{35}$ In the retrospective study of Nishijima revealed the prevalence of CAD was significantly higher in the major than non-major amputation group (82\% versus $63 \%, p=0.04)$. It also appeared that the risk of CAD was correlated with the severity of limb ischemia. ${ }^{36}$ These emphasized the need for continued monitoring and treatment of macrovascular and microvascular disease of all patients with diabetes, in particular, diabetes foot patients with documented CAD.

Severity classification based on Wagner was an important risk factor for LEA. $^{37}$ Our study confirmed that Wagner 5 increased major amputation risk more than 5 times. This observation was not surprising since the severity of the ulcer, including ulcer depth, osteomyelitis and necrosis/gangrene, increases with increasing Wagner grading.

APTT is the most commonly used sensitive indicator to reflect the coagulation activity of endogenous coagulation system. It can be used for monitoring unfractionated heparin therapy, as well as for screening lupus anticoagulant or for assessing thrombosis risk. There was no research on APTT as a risk factor of major amputation in patients with diabetic foot at present. Diabetes impaired the coagulation homeostasis which caused vascular thrombotic events. ${ }^{38}$ Bashir's study indicated that APTT value was significant in diabetic septic foot patients compared to non-diabetic septic foot patients $(32.64 \pm 5.2$ versus 28.49 $\pm 4.13, p<0.01$ ), and the author held that APTT was associated with prothrombotic state and hypercoagulability in patients with diabetic septic foot. ${ }^{39}$ Some diseases that may prolong APTT value, such as liver disease, vitamin $\mathrm{K}$ deficiency, sepsis, and disseminated intravascular coagulation and so on, were not found in the case data. We did the correlation test between APTT and other variables, and the results showed that $\mathrm{WBC}, \mathrm{Hb}, \mathrm{ALB}$ were correlated with APTT, which was statistically significant as shown in the figure below, but the correlation was slight $(r=0.155$, $r=0.252, r=0.247)$. In Teresa Cheng's study, the strongest correlation was found between the canine activated clotting time (ACT) and C-reactive protein (CRP) $(r=$ 0.66), a positive correlation between APTT and inflammation (assessed by CRP) was also recognized. Inflammation and coagulation both occur over a continuous spectrum of illness severity and the precise moment at which 
inflammation-induced activation of coagulation advances to systemic hypocoagulation is unknown. ${ }^{40}$ Therefore, we found APTT might be a newly discovered predictor of major amputation in patients with diabetic foot. This may be due to the severe systemic inflammation, poor nutritional status and hypercoagulable state, which subsequent increased of endogenous fibrinolysis in patients with major amputation group. Just as APTT may be a risk factor for major amputation, the reason and mechanism of this correlation need further experimental study. In addition, the values of D-dimer and Fib in major amputation group were higher than those in minor amputation group, however, there was no significant difference in multivariate analysis.

In multivariate analysis, baseline serum ALB and $\mathrm{Hb}$ levels were additional predictors of major amputation. Studies conducted by Aziz and Namgoong et al also found that the ALB and $\mathrm{Hb}$ level were significant prognostic factors for major amputation. ${ }^{41,42}$ Our study showed that serum ALB and $\mathrm{Hb}$ levels were negatively correlated with major amputation risk in diabetic foot patients. The higher the serum $\mathrm{Hb}$ level is, the more oxygen molecules are transported to local tissue. Serum ALB and $\mathrm{Hb}$ can also be used as indicators to evaluate the nutritional status of the human body. The wound consumed large quantities of energy during the healing process, therefore, low serum ALB and $\mathrm{Hb}$ levels will delay diabetic foot wound healing. This condition is definitely related to the high risk of major amputation in patients with diabetic foot.

WBC is an inflammatory markers for diabetic foot ulcers and other diseases and the level of WBC can reflect the severity of DFU infection. This study showed that higher baseline WBC levels predicted a higher probability of major amputation. Jiang et al study results also showed that increased WBC was associated with increased risks from DFU to major amputation in China ([OR] $1.1095 \%$ CI [1.02-1.19]). ${ }^{43}$ Other inflammatory markers, such as ESR, CRP and procalcitonin, may also be used as predictors of amputation, but this was still controversy. ${ }^{44}$ However, there is no doubt that early sensitive antiinfective treatment will benefit newly hospitalized patients with diabetic foot.

Our study had all the limitations inherent to retrospective studies. For example, patient compliance, which can affect the outcomes, was not considered. Our study population was from single center in China, and there can be a selection bias. Our hospital is a tertiary referral centre for complex diabetic foot ulcer, therefore, the results of this study might not be applicable to the general population or primary care centres.

\section{Conclusions}

The study found that major amputation was associated with previous amputation history, smoking, CAD, Wagner 5, ABI <0.4, HbA1c, Hb, ALB, WBC, and APTT might be a new risk factor. In conclusion, it is crucial importance to early identify the high-risk factors of major amputation in patients with diabetic foot, which can help determine prevention and treatment protocols when considering disease management, prioritization of treatment and health-related quality of life, and promptly orientated by a trained multidisciplinary team, which are essential to reduce the disability rate and family and society burden.

\section{Ethical Consideration}

Informed verbal consent was required from the patient in the study process, or required from the patient's next of kin because a few patients had passed away. The informed verbal consent process and the study were approved by the ethics committee of First Teaching Hospital of Tianjin University of Traditional Chinese Medicine (TYLL2017 [K] word 024). We promised that the data of the participants were anonymized or main tained with confidentiality and confirmed that the guide lines outlined in the Declaration of Helsinki were followed.

\section{Author Contributions}

All authors made substantial contributions to conception and design, acquisition of data, or analysis and interpretation of data; All authors took part in drafting the article or revising it critically for important intellectual content and agreed to submit to the current journal; All authors gave final approval of the version to be published and agree to be accountable for all aspects of the work.

\section{Funding}

This study was funded by the National Natural Science Foundation of China $(81973854,81473685)$ and Ministry of Science and Technology of China (2008BAI53B01, 2008BAI53B011).

\section{Disclosure}

The authors declare no conflicts of interest in this work. 


\section{References}

1. Armstrong DG, Boulton AJM, Bus SA. Diabetic foot ulcers and their recurrence. $N$ Engl J Med. 2017;376(24):2367-2375. doi:10.1056/ NEJMra1615439

2. IDF Diabetes Atlas. Ninth edition 2019. International Diabetes Federation; 2019. Available from: http://www.diabetesatlas.org. Accessed April 19, 2021.

3. Bowling FL, Rashid ST, Boulton AJ. Preventing and treating foot complications associated with diabetes mellitus. Nat Rev Endocrinol. 2015;11(10):606-616. doi:10.1038/nrendo.2015.130

4. Lu Q, Wang J, Wei X, et al. Cost of diabetic foot ulcer management in China: a 7-year single-center retrospective review. Diabetes Metab Syndr Obes. 2020;13:4249-4260. doi:10.2147/DMSO.S275814

5. Schaper NC, Van Netten JJ, Apelqvist J, et al. Prevention and management of foot problems in diabetes: a summary guidance for daily practice 2015, based on the IWGDF guidance documents. Diabetes Metab Res Rev. 2015;32(Suppl 1):7-15. doi:10.1002/dmrr.2695

6. van Netten JJ, Price PE, Lavery LA, et al. Prevention of foot ulcers in the at-risk patient with diabetes: a systematic review. Diabetes Metab Res Rev. 2016;32(Suppl 1):84-98. doi:10.1002/dmrr.2701

7. Bus SA, Waaijman R, Arts M, et al. Effect of custom-made footwear on foot ulcer recurrence in diabetes: a multicenter randomized controlled trial. Diabetes Care. 2013;36(12):4109-4116. doi:10.2337/ dc13-0996

8. Walsh JW, Hoffstad OJ, Sullivan MO, et al. Association of diabetic foot ulcer and death in a population-based cohort from the United Kingdom. Diabet Med. 2016;33(11):1493-1498. doi:10.1111/ dme. 13054

9. Iwase M, Fujii H, Nakamura U, et al. Incidence of diabetic foot ulcer in Japanese patients with type 2 diabetes mellitus: the Fukuoka diabetes registry. Diabetes Res Clin Pract. 2018;137:183-189. doi:10.1016/j.diabres.2018.01.020

10. Hurley L, Kelly L, Garrow AP, et al. A prospective study of risk factors for foot ulceration: the West of Ireland diabetes foot study. QJM. 2013;106(12):1103-1110. doi:10.1093/qjmed/hct182

11. Muller IS, de Grauw WJ, van Gerwen WH, et al. Foot ulceration and lower limb amputation in type 2 diabetic patients in dutch primary health care. Diabetes Care. 2002;25(3):570-574. doi:10.2337/ diacare.25.3.570

12. Lavery LA, Armstrong DG, Wunderlich RP, et al. Diabetic foot syndrome: evaluating the prevalence and incidence of foot pathology in Mexican Americans and non-Hispanic whites from a diabetes disease management cohort. Diabetes Care. 2003;26(5):1435-1438. doi:10.2337/diacare.26.5.1435

13. Jiang Y, Wang X, Xia L, et al. A cohort study of diabetic patients and diabetic foot ulceration patients in China. Wound Repair Regen. 2015;23(2):222-230. doi:10.1111/wrr. 12263

14. Edo AE, Edo GO, Ezeani IU. Risk factors, ulcer grade and management outcome of diabetic foot ulcers in a Tropical Tertiary Care Hospital. Niger Med J. 2013;54(1):59-63. doi:10.4103/03001652.108900

15. Sharath S, Henson H, Flynn S, et al. Ambulation and independence among veterans with nontraumatic bilateral lower-limb loss. $J$ Rehabil Res Dev. 2015;52(7):851-858. doi:10.1682/ JRRD.2014.07.0176

16. Nazri MY, Aminudin CA, Ahmad FS, et al. Quality of life of diabetes amputees following major and minor lower limb amputations. Med J Malaysia. 2019;74(1):25-29.

17. Uysal S, Arda B, Taşbakan MI, et al. Risk factors for amputation in patients with diabetic foot infection: a prospective study. Int Wound $J$. 2017;14(6):1219-1224. doi:10.1111/iwj.12788

18. Acar E, Kacira BK. Predictors of lower extremity amputation and reamputation associated with the diabetic foot. J Foot Ankle Surg. 2017;56(6):1218-1222. doi:10.1053/j.jfas.2017.06.004
19. Kurniawati A, Ismiarto YD, Hsu IL, et al. Prognostic factors for lower extremity amputation in diabetic foot ulcer patients. $J$ Acute Med. 2019;9(2):59-63. doi:10.6705/j.jacme.201906 9(2).0003

20. Ugwu E, Adeleye O, Gezawa I, et al. Predictors of lower extremity amputation in patients with diabetic foot ulcer: findings from MEDFUN, a multi-center observational study. $J$ Foot Ankle Res. 2019;12:34-42. doi:10.1186/s13047-019-0345-y

21. Kow RY, Low CL, Ruben JK, et al. Predictive factors of major lower extremity amputations in diabetic foot infections: a cross-sectional study at district hospital in Malaysia. Malays Orthop J. 2019;13 (3):45-52. doi:10.5704/MOJ.1911.008

22. Oyibo SO, Jude EB, Tarawneh I, et al. The effects of ulcer size and site, patient's age, sex and type and duration of diabetes on the outcome of diabetic foot ulcers. Diabet Med. 2001;18(2):133-138. doi:10.1046/j.1464-5491.2001.00422.x

23. Yesil S, Akinci B, Yener S, et al. Predictors of amputation in diabetics with foot ulcer: single center experience in a large Turkish cohort. Hormones. 2009;8(4):286-295. doi:10.14310/horm.2002.1245

24. Moon KC, Kim SB, Han SK, et al. Risk factors for major amputation in hospitalized diabetic patients with forefoot ulcers. Diabetes Res Clin Pract. 2019;158:107905. doi:10.1016/j.diabres.2019.107905

25. Adler AI, Erqou S, Lima TA, et al. Association between glycated haemoglobin and the risk of lower extremity amputation in patients with diabetes mellitus-review and meta-analysis. Diabetologia. 2010;53(5):840-849. doi:10.1007/s00125-009-1638-7

26. Miller W, Berg C, Wilson ML, et al. Risk factors for below-the-knee amputation in diabetic foot osteomyelitis after minor amputation. J Am Podiatr Med Assoc. 2019;109(2):91-97. doi:10.7547/16-143

27. Sen P, Demirdal T, Emir B. Meta-analysis of risk factors for amputation in diabetic foot infections. Diabetes Metab Res Rev. 2019;35(7): e3165. doi:10.1002/dmrr.3165

28. Liu M, Zhang W, Yan Z, et al. Smoking increases the risk of diabetic foot amputation: a meta-analysis. Exp Ther Med. 2018;15 (2):1680-1685. doi:10.3892/etm.2017.5538

29. Resnick HE, Carter EA, Lindsay R, et al. Relation of lower-extremity amputation to all-cause and cardiovascular disease mortality in American Indians: the strong heart study. Diabetes Care. 2004;27 (6):1286-1293. doi:10.2337/diacare.27.6.1286

30. Ikura K, Hanai K, Shinjyo T, et al. HDL cholesterol as a predictor for the incidence of lower extremity amputation and wound-related death in patients with diabetic foot ulcers. Atherosclerosis. 2015;239 (2):465-469. doi:10.1016/j.atherosclerosis.2015.02.006

31. Ries Z, Rungprai C, Harpole B, et al. Incidence, risk factors, and causes for thirty-day unplanned readmissions following primary lower-extremity amputation in patients with diabetes. $J$ Bone Joint Surg Am. 2015;97(21):1774-1780. doi:10.2106/JBJS.O.00449

32. Calle-Pascual AL, Garcia-Torre N, Moraga I, et al. Epidemiology of nontraumatic lower-extremity amputation in area 7, Madrid, between 1989 and 1999: a population-based study. Diabetes Care. 2001;24 (9):1686-1699. doi:10.2337/diacare.24.9.1686

33. Faglia E, Clerici G, Clerissi J, et al. Early and five-year amputation and survival rate of diabetic patients with critical limb ischemia: data of a cohort study of 564 patients. Eur J Vasc Endovasc Surg. 2006;32 (5):484-490. doi:10.1016/j.ejvs.2006.03.006

34. Jolissaint JS, Shah SK, Martin MC, et al. Risk prediction of 30-day mortality after lower extremity major amputation. J Vasc Surg. 2019;70(6):1868-1876. doi:10.1016/j.jvs.2019.03.036

35. Olesen KKW, Gyldenkerne C, Thim T, et al. Peripheral artery disease, lower limb revascularization, and amputation in diabetes patients with and without coronary artery disease: a cohort study from the Western Denmark heart registry. BMJ Open Diabetes Res Care. 2021;9(1):e001803. doi:10.1136/bmjdrc-2020-001803

36. Nishijima A, Yamamoto N, Yoshida R, et al. Coronary artery disease in patients with critical limb ischemia undergoing major amputation or not. Plast Reconstr Surg Glob Open. 2017;5(6):e1377. doi:10.1097/GOX.0000000000001377 
37. Sadriwala Q, Gedam B, Akhtar M. Risk factors of amputation in diabetic foot infections. Int Surg J. 2018;5(4):1399-1402. doi:10.18203/2349-2902.isj20181118

38. Soltani MM, Dayer MR, Ataie G, et al. Coagulation factors evaluation in NIDDM patients. Am J Biochem Mol Biol. 2011;1 (1):244-254. doi:10.3923/ajbmb.2011.244.254

39. Bashir BA, Ali MS. Hemostatic state augmented with platelet indices among Sudanese diabetic septic foot. BMC Hematol. 2018;18:11-17. doi:10.1186/s12878-018-0104-5

40. Collins PW, Macchiavello LI, Lewis SJ, et al. Global tests of hae-mostasis in critically ill patients with severe sepsis syndrome compared to controls. $\mathrm{Br} J$ Haematol. 2006;135(2):220-227. doi:10.1111/j.1365-2141.2006.06281.x
41. Namgoong S, Jung S, Han SK, et al. Risk factors for major amputation in hospitalised diabetic foot patients. Int Wound J. 2016;13(Suppl 1):13-19. doi:10.1111/iwj.12526

42. Aziz Z, Lin WK, Nather A, et al. Predictive factors for lower extremity amputations in diabetic foot infections. Diabet Foot Ankle. 2011;2:7463-7469. doi:10.3402/dfa.v2i0.7463

43. Jiang Y, Ran X, Jia L, et al. Epidemiology of type 2 diabetic foot problems and predictive factors for amputation in China. Int J Low Extrem Wounds. 2015;14(1):19-27. doi:10.1177/1534734614564867

44. Moon KC, Kim KB, Han SK, et al. Risk factors for major amputation on hindfoot ulcers in hospitalized diabetic patients. Adv Wound Care. 2019;8(5):177-185. doi:10.1089/wound.2018.0814

\section{Publish your work in this journal}

Diabetes, Metabolic Syndrome and Obesity: Targets and Therapy is an international, peer-reviewed open-access journal committed to the rapid publication of the latest laboratory and clinical findings in the fields of diabetes, metabolic syndrome and obesity research. Original research, review, case reports, hypothesis formation, expert opinion and commentaries are all considered for publication. The manuscript management system is completely online and includes a very quick and fair peer-review system, which is all easy to use. Visit http://www.dovepress.com/testimonials.php to read real quotes from published authors.

Submit your manuscript here: https://www.dovepress.com/diabetes-metabolic-syndrome-and-obesity-targets-and-therapy-journal 